\title{
Impact of parenting style and upbringing on menstrual stress in adolescent South Indian girls
}

\author{
Christy Vijay ${ }^{1}$, Naveen Ramesh ${ }^{2}$, Kavita Peter Gonsalves ${ }^{3 *}$
}

\begin{abstract}
${ }^{1}$ Department of Obstetrics and Gynecology, St. John's Medical College and Hospital, Bangalore, Karnataka, India ${ }^{2}$ Department of Community Health, St. John's Medical College, Bangalore, Karnataka, India

${ }^{3}$ Department of Obstetrics and Gynecology, St. Martha's Hospital, Bangalore, Karnataka, India
\end{abstract}

Received: 20 June 2020

Accepted: 03 August 2020

\author{
*Correspondence: \\ Dr. Kavita Peter Gonsalves, \\ E-mail: kavita12@gmail.com
}

Copyright: (c) the author(s), publisher and licensee Medip Academy. This is an open-access article distributed under the terms of the Creative Commons Attribution Non-Commercial License, which permits unrestricted non-commercial use, distribution, and reproduction in any medium, provided the original work is properly cited.

\begin{abstract}
Background: School absenteeism because of major symptoms like dysmenorrhea has ranged from $14 \%$ to $51 \%$ and this has affected the participation of school going girls in school related activities. In developing countries, adolescent girls face health issues due to socio-economic, environmental conditions and gender discrimination. The parenting style has been explained by Baumrind, which harbours a particular coping mechanism. This study aimed to determine the level of stress in adolescent girls during menstruation using one of these standardised scales. Objective of this study was to assess stress levels during menstrual period among adolescent girls, problems faced during menstrual period and parenting skill effect on menstrual stress.

Methods: This was a cross sectional study done on adolescent girls 13 to 19 years of age. The tool used in this study included demographic details, parenting style, (PSDQ scale), prevalence of stress (PSS). All the adolescent girls aged 13-19 years.

Results: The study participants 54 (24.9\%) felt stressed during the days of the menstrual period, $186(85.7 \%)$ regarded their mother as the major source for menstruation related health issues, $112(49.3 \%)$ did not get support from their family during the time of the menstrual period. Girls with parents who are very authoritarian 28 (31.5\%) and very involved 34 (34\%), had stress during the menstrual period.

Conclusions: Parents play a very important role during the growing phase of an adolescent girl. The communication between parents and children related to menstrual health issues is dependent on educational status of the parents and their knowledge about menstrual health.
\end{abstract}

Keywords: Menstruation, Parenting, Stress, Uninvolved

\section{INTRODUCTION}

Dysmenorrhea and menstrual problems show that an estimate of over 600 million hours are lost from work every year. ${ }^{2}$ School absenteeism or work absenteeism because of major symptoms like dysmenorrhea has ranged from $14 \%$ to $51 \%$ and this has affected the participation of school going girls in school related activities. $^{2-5}$ About $50 \%$ of these students miss school because of dysmenorrhea. ${ }^{1-3}$ Literature review focuses on menstrual symptoms pertaining only to primary pre- menstrual syndrome and not on mental health of adolescent girls during menstruation. ${ }^{1,6-8}$

In developing countries, adolescent girls face health issues due to socio-economic, environmental conditions and gender discrimination. ${ }^{8}$ Menstruation related health issues apart from being an economic burden, are also one of the most common causes of absenteeism and poor academic performance among young girls. ${ }^{8}$ Menstrual patterns can be affected by age, ethnicity, family history, smoking, physical activity, and dietary habits. Stress can 
be a major cause or contributor of various menstrual irregularities. ${ }^{8}$ Many researchers like Cohen et al deduced various scales to understand stress levels faced by individuals at a given point in time. ${ }^{9}$ This study aimed to determine the level of stress in adolescent girls during menstruation using one of these standardised scales.

Parents play an important role in the development of their child's mental and physical growth. The type of parenting style has been explained by Baumrind and other researchers, namely, Uninvolved, Authoritative, Authoritarian, Permissive parenting styles. ${ }^{10,11}$ Each style of parenting harbours a particular characteristic coping mechanism in the child to various situations in life. ${ }^{10,11}$ This study being one of the few studies, looked into the relation between type of parenting style and its influence on menstrual issues in adolescent girls.

The objectives of this study were to assess stress levels during menstrual period among adolescent girls in a rural area of Karnataka; to assess the problems faced during menstrual period by adolescent girls in a rural area of Karnataka; to assess the impact of parenting skill on adolescent girls and its effect on menstrual stress.

\section{METHODS}

This was a cross sectional study done on adolescent girls 13 to 19 years of age from two government schools in a rural village of South India, Karnataka. The study commenced after taking permission from respective school principals. The school staffs were informed about the results of the study and were enlightened on the possible interventions for various coping mechanism for stress. Ethical clearance was obtained from the institutional ethical committee.

The tool used in this study included four parts. First part includes demographic details of the student, next part looked into the various type of parenting style (uninvolved, authoritarian, authoritative, permissive) using a short version of the PSDQ scale. ${ }^{10,12}$ The short version of the PSDQ scale had 24 questions that looked into various parenting styles assessed by children who were the study girls. The third part measured the prevalence of stress as assessed by the perceived stressed scale (PSS). ${ }^{9}$ This was a five-point likert scale which ranged from a scoring of never (zero) to almost always (four). High score was indicative of high stress levels. ${ }^{13}$ Questions with regard to problems faced during menstrual cycle was assessed using semi-structured, open ended, face validated questionnaire.

All (universal sampling) the adolescents girls aged 13-19 years, studying in two government schools located in rural villages in Karnataka and those present on the day of the study were included. Assent/consent was taken from 217 girls who agreed to be part of this study.
The study questionnaire was distributed to all the girls after a brief introduction about the questionnaire. The completed questionnaire was collected by the authors. At the end of the session, the children witnessed an infotainment program on life stressors and its impact on mental health.

\section{Statistical analysis}

Data was analyzed using the SPSS versions 16 after it was manually entered in Microsoft excel. Inter-quartile ranges were deduced for the PSS and the PSDQ scale for analysis of stress levels and parenting style respectively. The Pearson's chi-square, Fishers exact and multi nominal regressions was done for significance.

\section{RESULTS}

This study involved 217 girls in the age group 13 to 19 years. Most of the girls were underweight (BMI <18.5) and belonged to the lower middle-class socio-economic status group (BG Prasad classification). ${ }^{15}$ Majority of the girl's had both parents in the age group of 31-41 years and most of their parents had completed secondary education.

Table 1, among the study participants 54 (24.9\%) felt stressed during the days of the menstrual period, 44 $(20.3 \%)$ responded that they were unable to get sanitary pads freely, $186(85.7 \%)$ regarded their mother as the major source for menstruation related health issues, majority availed sanitary pads from the shop $162(74.7 \%)$ and $24(11.1 \%)$ from anganwadi and $112(49.3 \%)$ did not get support from their family during the time of the menstrual period. The most common problem faced by adolescent girls was described by them was "a samayadhalli thumba ayasa waguthede" (which means "excessive fatigue during menstrual period").

Total (N) varies with each section, because some girls did not or were unable to answer those questions. $a=$ chisquare; b=Fisher's Exact; *statistically significant at $\alpha=5 \%$ and $p<0.05$.

M1: (response: yes); stress was found to be more among girls who were 14 years of age $14(35 \%)$, undernourished $18(19.4 \%)$, parents more than fifty years (father $50 \%$ and mother $100 \%$ ), parents with secondary education (father $24.2 \%$ and mother $24.8 \%$ ) and with occupational skill level two (father $28 \%$ and mother $27.3 \%$ ).

M2: (response: no); girls responded that they were not able to get sanitary pads freely and regularly. Girls who were underweight $21(22.6 \%)$, lower socioeconomic class $9(26.5 \%)$ had expressed their inability in availing sanitary pads. Girls with parents of skill level 1 (father $20.5 \%$ and mother $19 \%$ ) had also replied difficulty in receiving sanitary pads. 
M5: (response: no); stress was found to be more among girls who were 17 years of age $4(57.1 \%)$, overweight 4 (80\%), upper class $2(66.7 \%)$, parents more than forty years (father $60.3 \%$ and mother $70 \%$ ), parents with poor educational qualification (father $100 \%$ and mother $84 \%$ ) and with occupational skill level one (father $55.5 \%$ and mother $51.6 \%$ ).

Table 1: Stressors faced by adolescent girls during menstruation.

\begin{tabular}{|c|c|c|c|}
\hline Variables & & Frequency & Percentage \\
\hline \multirow{2}{*}{$\begin{array}{l}\text { M1: Do you feel stressed during the } \\
\text { days of the menstrual period? }\end{array}$} & No & 163 & 75.1 \\
\hline & Yes & 54 & 24.9 \\
\hline \multirow{2}{*}{$\begin{array}{l}\text { M2: Are you able to get sanitary pads } \\
\text { freely and regularly? }\end{array}$} & No & 44 & 20.3 \\
\hline & Yes & 173 & 79.7 \\
\hline \multirow{7}{*}{$\begin{array}{l}\text { M3: Who do you go to for help } \\
\text { regarding questions of menstrual } \\
\text { period? }\end{array}$} & No & 21 & 9.6 \\
\hline & Mother & 186 & 85.7 \\
\hline & Sister & 1 & 0.5 \\
\hline & Mother and sister & 3 & 1.4 \\
\hline & Sister and friend & 1 & 0.5 \\
\hline & Friend & 3 & 1.4 \\
\hline & All (mother, friend, sister media) & 2 & 0.9 \\
\hline \multirow{5}{*}{$\begin{array}{l}\text { M4: From where do you get sanitary } \\
\text { pads for use? }\end{array}$} & No & 20 & 9.2 \\
\hline & Shop & 162 & 74.7 \\
\hline & Anganwadi & 24 & 11.1 \\
\hline & Both & 6 & 28.8 \\
\hline & Others & 5 & 2.3 \\
\hline \multirow{3}{*}{$\begin{array}{l}\text { M5: Are you getting support from } \\
\text { your family? }\end{array}$} & No & 112 & 49.3 \\
\hline & Yes & 113 & 50.6 \\
\hline & Father & 2 & 0.9 \\
\hline \multirow{2}{*}{$\begin{array}{l}\text { M6: Are you getting time to take care } \\
\text { of yourself? }\end{array}$} & No & 111 & 51.2 \\
\hline & Yes & 106 & 48.8 \\
\hline \multirow{2}{*}{$\begin{array}{l}\text { M7: Do you face any problems during } \\
\text { the menstrual period? }\end{array}$} & No & 160 & 73.7 \\
\hline & Yes & 57 & 26.3 \\
\hline $\begin{array}{l}\text { M7: Do you face any problems during } \\
\text { the menstrual period? }\end{array}$ & $\begin{array}{l}\text { Descriptive: "a samayadhalli thumba } \\
\text { ayasawaguthede" was a common } \\
\text { complain of the girls of the schools } \\
\text { which was responded by many }\end{array}$ & & $\begin{array}{l}\text { And another } \\
\text { symptoms pain } \\
\text { abdomen stomach } \\
\text { pain, general pain }\end{array}$ \\
\hline
\end{tabular}

Description of stressors faced by adolescent girls during menstruation and responses to various situations during the menstrual cycle. The most common problem faced by adolescent girls was described by them as "a samayadhalli thumba ayasa waguthede" (which means "excessive fatigue during menstrual period").

M6: (response: no); stress was found to be more among girls who were 15 years of age $56(56 \%)$, overweight 3 $(60 \%)$, lower class $24(70.6 \%)$, parents more than fifty years (father $66.7 \%$ and mother $100 \%$ ), and with occupational skill level two (father $54 \%$ and mother $54.5 \%)$.

M7: (response: yes); stress was found to be more among girls who were 17 years of age $3(42.9 \%)$, BMI $>30$ $\mathrm{kg} / \mathrm{m}^{2} 1(50 \%)$, upper middle class $5(35.7 \%)$, parents more than thirty years (father $41.8 \%$ and mother $29.9 \%$ ), and with occupational skill level two (father 28 and mother $27.3 \%$ ).

Total (N) varies with each section, because some girls did not or were unable to answer those questions. $a=$ chisquare; $b=$ Fisher's Exact; *statistically significant at $\alpha=5 \%$ and $p<0.05$.
M1: (response: yes); girls with parents who are very authoritarian $28(31.5 \%)$ and very involved 34 (34\%), had stress during the menstrual period.

M2: (response: no); the level of stress of not receiving sanitary pads freely and regularly was mild $30(34.5 \%$, $\mathrm{p}<0.001)$. Girls with parents who were not involved 18 $(22.5 \%)$ did not receive sanitary pads freely and regularly. Parents with not authoritative type of parenting style had girls who did not receive sanitary pads freely and regularly $4(28.6 \%)$.

M5: (response: no); stress level are very severe 36 $(53.7 \%)$ when girls don't get support from their family during menstruation. Significant associations were seen in not authoritative $14(100 \%, \mathrm{p}=0.001)$, not authoritarian $15(100 \%, \mathrm{p}<0.001)$, not permissive $24(75 \%, \mathrm{p}=0.015)$ parenting style, who had girls who did not receive support from their family. 
Table 2: Contributing stressors in the menstrual cycle.

\begin{tabular}{|c|c|c|c|c|c|c|c|c|c|c|c|c|}
\hline Variables & & $\begin{array}{l}\text { Total } \\
\mathbf{N}=217 \\
\mathbf{N}(\%)\end{array}$ & M1: Yes & $\begin{array}{l}P \\
\text { value }\end{array}$ & M2: No & $\begin{array}{l}\mathbf{P} \\
\text { value }\end{array}$ & M5: No & $\begin{array}{l}P \\
\text { value }\end{array}$ & M6: No & P value & M7: Yes & $\begin{array}{l}\mathbf{P} \\
\text { value }\end{array}$ \\
\hline \multirow{6}{*}{ Age } & 13 years & $8(100)$ & $2(25)$ & \multirow{6}{*}{$0.443^{b}$} & $2(25)$ & \multirow{6}{*}{$0.085^{\mathrm{b}}$} & $3(37.5)$ & \multirow{6}{*}{$0.805^{\mathrm{b}}$} & $4(50)$ & \multirow{6}{*}{$0.661^{b}$} & $2(25)$ & \multirow{6}{*}{$0.829^{b}$} \\
\hline & 14 years & $40(100)$ & $14(35)$ & & $8(20)$ & & $21(52.5)$ & & $21(52.5)$ & & $12(30)$ & \\
\hline & 15 years & $100(100)$ & $26(26)$ & & $27(27)$ & & $55(55)$ & & $56(56)$ & & $24(24)$ & \\
\hline & 16 years & $61(100)$ & $11(18)$ & & $7(11.5)$ & & $29(47.5$ & & $27(44.3)$ & & $16(26.2)$ & \\
\hline & 17 years & $7(100)$ & $1(14.3)$ & & $0(0)$ & & $4(57.1$ & & $3(42.9)$ & & $3(42.9)$ & \\
\hline & 19 years & $1(100)$ & $0(0)$ & & $0(0)$ & & $0(0)$ & & $0(0)$ & & $0(0)$ & \\
\hline \multirow{6}{*}{$\begin{array}{l}\text { Body mass } \\
\text { index } \\
\text { (Kg/meter } \\
\text { square) } \text { ) }^{14} \\
\text { (WHO) }\end{array}$} & $\begin{array}{l}\text { Underweight } \\
(<18.5)\end{array}$ & $93(100)$ & 18 (19.4) & \multirow{6}{*}{$0.104^{b}$} & $21(22.6)$ & \multirow{6}{*}{$0.194^{b}$} & $57(61.3)$ & \multirow{6}{*}{$0.025^{\mathrm{b}}$} & $54(58.1)$ & \multirow{6}{*}{$0.345^{b}$} & 25 (26.9) & \multirow{6}{*}{$0.705^{\mathrm{b}}$} \\
\hline & $\begin{array}{l}\text { Normal } \\
(18.5-24.9)\end{array}$ & $54(100)$ & $9(16.7)$ & & $5(9.3)$ & & $24(44.4)$ & & $25(46.3)$ & & 17 (31.5) & \\
\hline & $\begin{array}{l}\text { Overweight } \\
(25.0-29.9)\end{array}$ & $5(100)$ & $1(20)$ & & $1(20)$ & & $4(80)$ & & $3(60)$ & & $2(40)$ & \\
\hline & $\begin{array}{l}\text { Class I obesity } \\
(30.0-34.9)\end{array}$ & $2(100)$ & $0(0)$ & & $0(0)$ & & $0(0)$ & & $0(0)$ & & $1(50)$ & \\
\hline & $\begin{array}{l}\text { Class II obesity } \\
(35.0-39.9)\end{array}$ & $0(0)$ & $0(0)$ & & $0(0)$ & & $0(0)$ & & $0(0)$ & & $0(0)$ & \\
\hline & $\begin{array}{l}\text { Class III obesity } \\
(>40.0)\end{array}$ & $2(100)$ & $2(100)$ & & $0(0)$ & & $0(0)$ & & $1(50)$ & & $0(0)$ & \\
\hline \multirow{5}{*}{$\begin{array}{l}\text { Socio-economic } \\
\text { status } \\
\text { (modified BG } \\
\text { Prasad } \\
\text { classification) } \\
\text { All India } \\
2014 \\
\text { standards }^{15}\end{array}$} & $\begin{array}{l}\text { Lower class } \\
(<811)\end{array}$ & $34(100)$ & 7 (20.6) & \multirow{5}{*}{$0.624^{b}$} & $9(26.5)$ & \multirow{5}{*}{$0.754^{\mathrm{b}}$} & $20(58.8)$ & \multirow{5}{*}{$0.967^{\mathrm{b}}$} & $24(70.6)$ & \multirow{5}{*}{$0.414^{\mathrm{b}}$} & $9(26.5)$ & \multirow{5}{*}{$0.634^{b}$} \\
\hline & $\begin{array}{l}\text { Lower middle } \\
\text { class }(812-1569)\end{array}$ & $70(100)$ & $19(27.1)$ & & $18(25.7)$ & & $41(58.6)$ & & 37 (52.9) & & $14(20)$ & \\
\hline & $\begin{array}{l}\text { Middle class } \\
(1570-2651)\end{array}$ & $34(100)$ & 7 (20.6) & & $8(23.5)$ & & $18(52.9)$ & & $17(50)$ & & $9(26.5)$ & \\
\hline & $\begin{array}{l}\text { Upper middle class } \\
(2652-5356)\end{array}$ & $14(100)$ & $4(28.6)$ & & $3(21.4)$ & & $9(64.3)$ & & $8(57.1)$ & & $5(35.7)$ & \\
\hline & $\begin{array}{l}\text { Upper class } \\
(>5357)\end{array}$ & $3(100)$ & $0(0)$ & & $0(0)$ & & $2(66.7)$ & & $2(66.7)$ & & $0(0)$ & \\
\hline \multirow{4}{*}{ Father's age } & $<30$ years & $4(100)$ & $0(0)$ & \multirow{4}{*}{$0.177^{b}$} & $0(0)$ & \multirow{4}{*}{$0.537^{b}$} & $2(50)$ & & $2(50)$ & \multirow{4}{*}{$0.000^{\mathrm{b}}$} & $0(0)$ & \multirow[t]{4}{*}{$0.010^{\mathrm{b}}$} \\
\hline & $31-40$ years & $67(100)$ & $17(24.4)$ & & $12(17.9)$ & & $34(50.7)$ & & $40(59.7)$ & & $28(41.8)$ & \\
\hline & $41-50$ years & $58(100)$ & $11(19.0)$ & & $13(22.4)$ & & $35(60.3)$ & $0.748^{\mathrm{b}}$ & $34(58.6)$ & & $12(20.7)$ & \\
\hline & $50-60$ years & $6(100)$ & $3(50.0)$ & & $1(16.7)$ & & $3(50)$ & & $4(66.7)$ & & $0(0)$ & \\
\hline
\end{tabular}




\begin{tabular}{|c|c|c|c|c|c|c|c|c|c|c|c|c|}
\hline Variables & & $\begin{array}{l}\text { Total } \\
\mathbf{N}=217 \\
\mathbf{N}(\%)\end{array}$ & M1: Yes & $\begin{array}{l}P \\
\text { value }\end{array}$ & M2: No & $\begin{array}{l}\mathbf{P} \\
\text { value }\end{array}$ & M5: No & $\begin{array}{l}\mathbf{P} \\
\text { value }\end{array}$ & M6: No & P value & M7: Yes & $\begin{array}{l}\mathbf{P} \\
\text { value }\end{array}$ \\
\hline \multirow{4}{*}{ Mother's age } & $<30$ years & $32(100)$ & $9(28.1)$ & \multirow{4}{*}{$0.416^{\mathrm{b}}$} & $4(12.5)$ & \multirow{4}{*}{$0.290^{\mathrm{b}}$} & $12(37.5)$ & \multirow{4}{*}{$0.060^{\mathrm{b}}$} & $13(40.6)$ & \multirow{4}{*}{$0.076^{\mathrm{b}}$} & $9(28.1)$ & \multirow{4}{*}{$0.634^{\mathrm{B}}$} \\
\hline & $31-40$ years & $107(100)$ & $27(25.2)$ & & $20(18.7)$ & & $63(58.9)$ & & $67(62.6)$ & & $32(29.9)$ & \\
\hline & $41-50$ years & $\begin{array}{l}10 \\
(100)\end{array}$ & $3(30)$ & & $4(40.0)$ & & $7(70)$ & & $7(70)$ & & $1(10)$ & \\
\hline & $50-60$ years & $1(100)$ & $1(100)$ & & $0(0)$ & & $0(0)$ & & $1(100)$ & & $0(0)$ & \\
\hline \multirow{5}{*}{$\begin{array}{l}\text { Father's } \\
\text { education }\end{array}$} & Illiterate & $7(100)$ & $0(0)$ & \multirow{5}{*}{$0.066^{\mathrm{b}}$} & $0(0)$ & \multirow{5}{*}{$0.084^{\mathrm{b}}$} & $7(100)$ & \multirow{5}{*}{$0.041^{\mathrm{b}}$} & $6(85.7)$ & \multirow{5}{*}{$0.230^{\mathrm{b}}$} & $2(28.6)$ & \multirow{5}{*}{$0.897^{\mathrm{b}}$} \\
\hline & $\begin{array}{l}\text { Primary Education } \\
\left(1-4^{\text {th }} \text { standard }\right)\end{array}$ & $16(100)$ & $8(50)$ & & $6(37.5)$ & & $9(56.2)$ & & $6(37.5)$ & & $6(37.5)$ & \\
\hline & $\begin{array}{l}\text { Secondary Educatior } \\
5-10^{\text {th }} \text { standard) }\end{array}$ & $124(100)$ & $30(24.2)$ & & $\begin{array}{l}22 \\
(17.7)\end{array}$ & & $61(49.2)$ & & $70(56.5)$ & & $34(27.4)$ & \\
\hline & $\begin{array}{l}\text { Pre- Graduation } \\
11-12^{\text {th }} \text { standard/ } \\
\text { pre university) }\end{array}$ & $10(100)$ & $3(30)$ & & $4(40)$ & & $5(50)$ & & $4(40)$ & & $2(20)$ & \\
\hline & $\begin{array}{l}\text { Graduation } \\
\left(>12^{\text {th }} \text { standard }\right)\end{array}$ & $5(100)$ & $1(28)$ & & $1(20)$ & & $2(40)$ & & $3(60)$ & & $1(20)$ & \\
\hline \multirow{5}{*}{$\begin{array}{l}\text { Mother's } \\
\text { education }\end{array}$} & Illiterate & $0(0)$ & $0(0)$ & \multirow{5}{*}{$0.408^{\mathrm{b}}$} & & \multirow{5}{*}{$0.133^{\mathrm{b}}$} & $0(0)$ & \multirow{5}{*}{$0.303^{b}$} & $0(0)$ & \multirow{5}{*}{$0.850^{\mathrm{b}}$} & & \multirow{5}{*}{$0.622^{\mathrm{b}}$} \\
\hline & $\begin{array}{l}\text { Primary } \\
\text { education } \\
\left(1-4^{\text {th }} \text { standard }\right)\end{array}$ & $25(100)$ & $10(40)$ & & $5(20)$ & & $21(84)$ & & $13(52)$ & & $4(16)$ & \\
\hline & $\begin{array}{l}\text { Secondary } \\
\text { education } \\
5-10^{\text {th }} \text { standard) }\end{array}$ & $113(100)$ & $28(24.8)$ & & $\begin{array}{l}27 \\
(23.9)\end{array}$ & & $\begin{array}{l}82 \\
(72.6)\end{array}$ & & $62(54.9)$ & & $31(27.4)$ & \\
\hline & $\begin{array}{l}\text { Pre- Graduation } \\
11-12^{\text {th }} \text { standard/pre } \\
\text { university) }\end{array}$ & $14(100)$ & $4(28.6)$ & & $1(7.1)$ & & $10(71.4)$ & & $9(64.3)$ & & $4(28.6)$ & \\
\hline & $\begin{array}{l}\text { Graduation } \\
\left(>12^{\text {th }} \text { standard }\right)\end{array}$ & $1(100)$ & $0(0)$ & & $1(100)$ & & $1(100)$ & & $1(100)$ & & $0(0)$ & \\
\hline \multirow{2}{*}{$\begin{array}{l}\text { Occupation of } \\
\text { father } \\
(\text { ISCO-08) })^{16}\end{array}$} & Skill level 1 & $146(100)$ & $36(24.7)$ & \multirow[t]{2}{*}{$0.640^{\mathrm{a}}$} & $\begin{array}{l}30 \\
(20.5)\end{array}$ & \multirow[t]{2}{*}{$0.483^{\mathrm{a}}$} & $\begin{array}{l}81 \\
(55.5)\end{array}$ & \multirow[t]{2}{*}{$0.161^{\mathrm{a}}$} & $74(50.7)$ & \multirow[t]{2}{*}{$0.686^{\mathrm{a}}$} & $40(27.4)$ & \multirow[t]{2}{*}{$0.453^{\mathrm{a}}$} \\
\hline & Skill level 2 & $50(100)$ & $14(28)$ & & $8(16)$ & & $22(44)$ & & $27(54)$ & & $11(22)$ & \\
\hline \multirow{2}{*}{$\begin{array}{l}\text { Occupation of } \\
\text { mother } \\
(\text { ISCO-08) }\end{array}$} & Skill level 1 & $184(100)$ & $38(20.7)$ & \multirow[b]{2}{*}{$0.612^{\mathrm{b}}$} & $35(19)$ & \multirow[b]{2}{*}{$1.000^{\mathrm{b}}$} & $95(51.6)$ & \multirow[b]{2}{*}{$0.325^{\mathrm{a}}$} & $92(50)$ & \multirow[b]{2}{*}{$0.770^{\mathrm{a}}$} & $52(28.3)$ & \multirow[b]{2}{*}{$0.516^{\mathrm{b}}$} \\
\hline & Skill level 2 & $11(100)$ & $3(27.3)$ & & $2(18.2)$ & & $4(36.4)$ & & $6(54.5)$ & & $4(36.4)$ & \\
\hline
\end{tabular}

Total $(\mathrm{N})$ varies with each section, because some girls did not or were unable to answer those questions. a=Chi-Square; $b=$ Fisher's exact; *statistically significant at $\alpha=5 \%$ and $p<0.05$. Factors that contributed to the overall stress levels and underlying causes for the same. Responses are indicative of the respective factor namely, M1: (response: yes); M2: (response: no); M5: (response: no); M6: (response: no); M7: (response: yes). 
Table 3: Stressors faced by adolescent girls during menstruation and parental style.

\begin{tabular}{|c|c|c|c|c|c|c|c|c|c|c|c|c|}
\hline Variables & & $\begin{array}{l}\text { Total } \\
\mathbf{N}=217 \\
\mathbf{N}(\%)\end{array}$ & $\begin{array}{l}\text { M1: } \\
\text { Yes }\end{array}$ & $\begin{array}{l}P \\
\text { value }\end{array}$ & $\begin{array}{l}\text { M2: } \\
\text { No }\end{array}$ & $\begin{array}{l}P \\
\text { value }\end{array}$ & $\begin{array}{l}\text { M5: } \\
\text { No }\end{array}$ & $\begin{array}{l}\mathbf{P} \\
\text { value }\end{array}$ & $\begin{array}{l}\text { M6: } \\
\text { No }\end{array}$ & $\begin{array}{l}P \\
\text { value }\end{array}$ & $\begin{array}{l}\text { M7: } \\
\text { Yes }\end{array}$ & $\begin{array}{l}P \\
\text { value }\end{array}$ \\
\hline \multirow{3}{*}{ PSS quartiles ${ }^{9}$} & Mild stress & 87 (100) & $27(31)$ & \multirow{3}{*}{$0.065^{\mathrm{a}}$} & $30(34.5)$ & \multirow{3}{*}{$0.000^{\mathrm{a}}$} & $46(52.9)$ & \multirow{3}{*}{$0.749^{\mathrm{a}}$} & $52(59.8)$ & \multirow{3}{*}{$0.114^{\mathrm{a}}$} & $24(27.6)$ & \multirow{3}{*}{$0.460^{\mathrm{a}}$} \\
\hline & Moderate stress & $63(100)$ & $17(27)$ & & $10(15.9)$ & & $30(47.6)$ & & $29(46)$ & & $13(20.6)$ & \\
\hline & Severe stress & $67(100)$ & $10(14.9)$ & & $4(6.0)$ & & $36(53.7)$ & & $30(44.8)$ & & $20(29.9)$ & \\
\hline \multirow{3}{*}{$\begin{array}{l}\text { Domain 1 } \\
\text { uninvolved } \\
\text { parenting } \\
\text { style }{ }^{10,11}\end{array}$} & Very involved & $100(100)$ & $34(34)$ & \multirow{3}{*}{$0.016^{\mathrm{a}}$} & $21(21)$ & & $56(56)$ & \multirow{3}{*}{$0.053^{\mathrm{a}}$} & $50(50)$ & \multirow{3}{*}{$0.533^{\mathrm{a}}$} & $23(23)$ & \multirow{3}{*}{$0.066^{\circ}$} \\
\hline & $\begin{array}{l}\text { Moderate } \\
\text { involved }\end{array}$ & 37 (100) & $6(16.2)$ & & $5(13.5)$ & $0.516^{\mathrm{b}}$ & $23(62.2)$ & & $22(59.6)$ & & $6(16.2)$ & \\
\hline & Not involved & $80(100)$ & $14(17.5)$ & & $18(22.5)$ & & $33(41.2)$ & & $39(48.8)$ & & $28(35)$ & \\
\hline \multirow{3}{*}{$\begin{array}{l}\text { Domain } 2 \\
\text { authoritative } \\
\text { parenting } \\
\text { style }^{\mathbf{1 0 , 1 1}}\end{array}$} & $\begin{array}{l}\text { Not } \\
\text { authoritative }\end{array}$ & $14(100)$ & $2(14.3)$ & \multirow{3}{*}{$0.150^{\mathrm{b}}$} & 4 (28.6) & \multirow{3}{*}{$0.638^{\mathrm{b}}$} & $14(100)$ & \multirow{3}{*}{$0.001^{\mathrm{a}}$} & $6(42.9)$ & \multirow{3}{*}{$0.563^{\mathrm{a}}$} & $0(0)$ & \multirow{3}{*}{$0.045^{\mathrm{b}}$} \\
\hline & $\begin{array}{l}\text { Moderate } \\
\text { authoritative }\end{array}$ & $89(100)$ & $28(31.5)$ & & $16(18)$ & & $43(48.3)$ & & $43(48.3)$ & & $24(27)$ & \\
\hline & $\begin{array}{l}\text { Very } \\
\text { authoritative }\end{array}$ & $114(100)$ & $24(21.1)$ & & $24(21.1)$ & & $55(48.2)$ & & $62(54.4)$ & & 33 (28.9) & \\
\hline \multirow{3}{*}{$\begin{array}{l}\text { Domain } 3 \\
\text { Authoritarian } \\
\text { parenting } \\
\text { style }^{\mathbf{1 0 , 1 1}}\end{array}$} & $\begin{array}{l}\text { Not } \\
\text { authoritarian }\end{array}$ & $15(100)$ & $1(6.7)$ & \multirow{3}{*}{$0.053^{\mathrm{b}}$} & $3(20)$ & \multirow{3}{*}{$0.045^{\mathrm{b}}$} & $15(100)$ & \multirow{3}{*}{$0.000^{\mathrm{a}}$} & $4(26.7)$ & \multirow{3}{*}{$0.23^{\mathrm{a}}$} & $1(6.7)$ & \multirow{3}{*}{$0.075^{\mathrm{b}}$} \\
\hline & $\begin{array}{l}\text { Moderate } \\
\text { authoritarian }\end{array}$ & $113(100)$ & $25(22.1)$ & & $30(26.5)$ & & $58(51.3)$ & & $64(56.6)$ & & $27(23.9)$ & \\
\hline & $\begin{array}{l}\text { Very } \\
\text { authoritarian }\end{array}$ & $89(100)$ & $28(31.5)$ & & $11(12.4)$ & & $39(43.8)$ & & $43(48.3)$ & & $29(32.6)$ & \\
\hline \multirow{3}{*}{$\begin{array}{l}\text { Domain } 4 \\
\text { permissive } \\
\text { parenting } \\
\text { style }^{10,11}\end{array}$} & Not permissive & $32(100)$ & $11(34.4)$ & \multirow{3}{*}{$0.033^{\mathrm{a}}$} & $12(37.5)$ & \multirow{3}{*}{$0.029^{\mathrm{a}}$} & $24(75)$ & \multirow{3}{*}{$0.015^{\mathrm{a}}$} & $12(37.5)$ & \multirow{3}{*}{$0.206^{\mathrm{a}}$} & $6(18.18)$ & \multirow{3}{*}{$0.511^{\mathrm{a}}$} \\
\hline & $\begin{array}{l}\text { Moderate } \\
\text { permissive }\end{array}$ & $133(100)$ & $25(18.8)$ & & $22(16.5)$ & & $62(46.6)$ & & $73(54.9)$ & & $38(28.6)$ & \\
\hline & $\begin{array}{l}\text { Very } \\
\text { permissive }\end{array}$ & $52(100)$ & $18(34.6)$ & & $10(19.2)$ & & $26(50)$ & & $26(50)$ & & $13(25)$ & \\
\hline
\end{tabular}

Total $(\mathrm{N})$ varies with each section, because some girls did not or were unable to answer those questions. $\mathrm{a}=$ Chi-square; $\mathrm{b}=$ Fisher's exact; *statistically significant at $\alpha=5 \%$ and $\mathrm{p}<0.05$. Description of stressors faced by Adolescent girls during Menstruation in relation to parental style of upbringing and its influence on overall stress faced during the menstrual cycle. Responses are indicative of the respective factor namely, M1: (response: yes); M2: (response: no); M5: (response: no); M6: (response: no); M7: (response: yes). 
M6: (response: no); The level of stress was overall mild $52(59.8 \%)$. Very authoritative $62(54.4 \%)$ type of parenting style was associated with girls who did not receive time to take care of themselves during menstruation.

M7: (response: yes); Not involved 28 (35\%), very authoritarian 29 (32.6\%), and very authoritative 33 $(28.9 \%, p=0.045)$ type of parenting style showed girls to face more complaints during the menstrual period.

\section{DISCUSSION}

Menstrual disorders affect health and increase the financial burden on society worldwide. A major gynecologic complaint leading to school absenteeism is dysmenorrhea. ${ }^{1,3,17}$ This study looked into menstrual stress levels in adolescent rural girls and the impact parenting had on coping with this stress. Smetana et al looked into parenting styles, dimensions and beliefs. ${ }^{18}$ The original authoritative, authoritarian and permissive parenting styles was conceptualized and involved a fourth dimension of uninvolved style. ${ }^{18}$ This study looked into which of these parenting styles would affect adolescent girls during the menstrual period.

Girls who had very involved parents $(34 \%, \mathrm{p}=0.016)$ felt stressed during menstruation, probably due to their parents over concerned attitude of the same. Very authoritarian $(31.5 \%)$ parenting styles showed girls to be stressed during menstruation. This style is common in non-western cultures and has a protective effect on adolescents. ${ }^{18,19}$ Studies have shown children to have adjustment issues with this parenting style although they are culturally acceptable. ${ }^{18,20-22}$ This has been tested in this study showing an inverse relation.

Very authoritative $(54.4 \%)$ parenting, showed girls felt that they did not get enough time to take care of themselves during menstruation, the possibility being the need to be more academically inclined during this phase of growing, causing them to neglect menstrual problems. Girls who did not get support from their family during the menstrual period had not authoritative $(100 \%, \mathrm{p}=0.001)$, not authoritarian $(100 \%, \mathrm{p}<0.001)$, not permissive $(75 \%$, $\mathrm{p}=0.015$ ) type of parents, which is a well-adjusted type of parenting style for an Indian culture. The possibility of these girls internalizing and externalizing their problems is the outcome in this type of parenting (authoritarian rule), which is a harsh guilt induction parenting. ${ }^{18}$ In this type of parenting, parents wish to know if their adolescents are involved in dangerous activities. ${ }^{18,23}$

The overall stress faced by these adolescent girls was mild stress (PSS). ${ }^{9}$ The stress level was found to be severe $(29.9 \%)$ when it came to problems faced during the menstrual period and not getting support from family members during the menstrual period (53.7\%). This probably shows that rural adolescent girls have high coping mechanism and are strong willed. It also supports the reasoning of Indian families needing support which could decrease problems on mental health when it comes to menstrual stress. The DASS-21 accessed the association of stress and menstrual signs and showed $47.2 \%$ has stress, similar to this study. ${ }^{24} \mathrm{~A}$ study in Pune showed moderate stress in $46.6 \%$, and $43.3 \%$ to have low stress and $10.1 \%$ to have high stress during menstruation, similar to this study. ${ }^{25}$

Agarwal et al showed source of information regarding menstruation was from mothers $(41.61 \%)$, sisters $(12.41 \%)$ and friends $(13.87 \%){ }^{26}$ In this study mothers $(85.7 \%)$ were the major source of information followed by friends $(1.4 \%)$ and sisters $(0.5 \%)$, similar findings were seen by Kamath et al. ${ }^{27}$

Main complaints faced in this study participants was fatigue, followed by abdominal discomfort and pain, which compared with a Indian study showed pain abdomen $(59.70 \%)$ to be the major complain followed by headache $(23.57 \%)$ and loss of appetite $(11.79 \%) .{ }^{26}$ Other studies done in West Bengal (66.9\%) and Garhwal $(62.75 \%)$ stated that dysmenorrhea was the most common complaint faced by adolescent girls. ${ }^{28,29}$

This study noticed low utilization of sanitary pads from the anganwadi $(11.1 \%)$. One study suggested the possibility of poor ASHA social marketing scheme implementation, needing the $\mathrm{NRHM}+\mathrm{A}$ to market the availability of sanitary pads in Anganwadi by ASHA workers. ${ }^{30}$ Girls above 15 years felt they did not receive support from their family and faced problems during the menstrual period, as care in an Indian society mostly goes to the younger child.

Girls with low BMI felt stressed (19.4\%), faced lesser complaints during the menstrual period $(26.9 \%)$ leading to the possibility of hormonal imbalances that exists in low nutritional state. Low SES groups had issues in getting sanitary pads freely and regularly (26.5\%), inability to take care of themselves $(70.6 \%)$, faced more problems $(73.5 \%)$ and got less support from their family (58.8\%) during the menstrual period, due to low resources at home and neglect. Another outlook to this could be low income families have parents working double shift to make ends meet leading to inability to spend time with their daughters as they need to sustain the family.

Girls who had fathers of an older age group (66.7\%), had no time to take care of themselves during the menstrual period, in comparison with girls with fathers of younger age group $(50 \%) \quad(\mathrm{p}<0.001)$, probability of change in paternal parenting style from authoritarian to permissive in a developing country. Girls with fathers educated to graduate level $(60 \%)$ had daughters who received good support from their family during the menstrual cycle $(p=0.041)$. This could be awareness of parents to be supportive to their daughters during the menstrual period 
showing a new trend in modern society where fathers are involved in menstrual care.

Limitations of this study was post intervention analysis on stress levels could have been done.

\section{CONCLUSION}

Parents play a very important role during the growing phase of an adolescent girl. The communication between parents and children related to menstrual health issues is dependent on educational status, of the parents and their knowledge about menstrual health. Mothers play a vital role and hence it's important that they be armed with appropriate information and knowledge about reproductive health. It is essential for the teachers to impart knowledge on reproductive health. Integrating menstrual hygiene into curriculum, provision of toilets and even supplying sanitary napkin at schools is the need of the hour especially in rural areas of India. Introducing stress management programmes in schools would create a huge impact on adolescents in dealing with not only health related stress but also mental stress. ASHAs along with village health and sanitation committee and local bodies must be oriented towards menstrual hygiene practices and help spread the awareness through IPC. Professional bodies like IAP and NGOs like UNICEF, UNFPA and PHFI should actively promote menstrual hygiene for better practices in the community.

\section{Recommendations}

National programmes focusing on mental health in the $\mathrm{NRHM}+\mathrm{A}$ scheme is warranted. This study was the first of its kind to see the association of the perceived stress scale with type of parenting skill of the parents and its effect on mental health in the adolescents

\section{ACKNOWLEDGMENTS}

Authors would like to thank the teachers of the Government schools of Masthi and Kesereger. The study team thanks Rev. Fr. Sibi, Dr. Manu, Sr. Sujatha, Sr. Neviditha, Mr. Amaresh and Vanaprastha Charitable Trust Rural Hospital and staff for their unconditional during this research.

\section{Funding: No funding sources}

Conflict of interest: None declared

Ethical approval: The study was approved by the Institutional Ethics Committee

\section{REFERENCES}

1. Dorn LD, Negriff S, Huang B, Pabst S, Hillman J, Braverman P, et al. Menstrual symptoms in adolescent girls: association with smoking, depressive symptoms, and anxiety. J Adolescent Health. 2009;44(3):237-43.
2. Dawood MY. Dysmenorrhea. J Reprod Med. 1985;30(3):154-67.

3. Klein JR, Litt IF. Epidemiology of adolescent dysmenorrhea. Pediatr. 1381;68(5):661-4.

4. Sundell G, Milsom I, Andersch B. Factors influencing the prevalence and severity of dysmenorrhoea in young women. $\mathrm{Br} \mathrm{J}$ Obstet Gynaecol. 1990;97(7):588-94.

5. Banikarim C, Chacko MR, Kelder SH. Prevalence and impact of dysmenorrhea on hispanic female adolescents. Am Medi Associat. 2000;154(12):1226.

6. Endicott J. History, evolution, and diagnosis of premenstrual dysphoric disorder. J Clin Psychiatr. 2000;61(Suppl 12):5-8.

7. Ross LE, Steiner M. A biopsychosocial approach to premenstrual dysphoric disorder. Psych Clin North Am. 2003;26(3):529-46.

8. Vichnin M, Freeman EW, Lin H, Hillman J, Bui S. Premenstrual syndrome (PMS) in adolescents: severity and impairment. J Pediatr Adolescent Gynecol. 2006;19(6):397-402.

9. Cohen S. Perceived stress scale (taken from a global measure of perceived stress). J Health Social Behav. 1983;24:386-96.

10. Tagliabue S, Olivari MG, Bacchini D, Affuso G, Confalonieri E. Measuring adolescents' perceptions of parenting style during childhood: psychometric properties of the parenting styles and dimensions questionnaire. Psicologia: Teoria e Pesquisa. 2014;30(3):251-8.

11. Blakely Kimble AB. The parenting styles and dimensions questionnaire: a reconceptualization and validation (doctoral dissertation, thesis approved by Hubbs-Tait L, submitted to the Faculty of the Graduate College of the Oklahoma State University. Stillwater: Oklahoma State University); 2014.

12. Johnson R, Welk G, Saint-Maurice PF, Ihmels M. Parenting styles and home obesogenic environments. Int J Environment Res Public Health. 2012;9(4):1411-26.

13. Terzian M, Moore KA, Nguyen HN. Assessing stress in children and youth: a guide for out- of-school time program practitioners, Research-to-Results; 2010.

14. World Health Organization. Global database on body mass index. 2006. Global Database on Body Mass Index. 2016.

15. Mangal A, Kumar V, Panesar S, Talwar R, Raut D, Singh S. Updated BG Prasad socioeconomic classification, 2014: a commentary. Indian J Public Health. 2015;59(1):42.

16. Uter W. Classification of occupations, in Kanerva's occupational dermatology, Second Edition. Springer Berlin Heidelberg; 2012:45-50.

17. Hillen TI, Grbavac SL, Johnston PJ, Straton JA, Keogh JM. Primary dysmenorrhea in young Western Australian women: prevalence, impact, and knowledge of treatment. J Adolescent Health. 1999;25(1):40-5. 
18. Smetana JG. Current research on parenting styles, dimensions, and beliefs. Current Opin Psychol. 2017; $15: 19-25$.

19. Lee EH, Zhou Q, Ly J, Main A, Tao A, Chen SH. Neighborhood characteristics, parenting styles, and children's behavioral problems in Chinese American immigrant families. Cul Divers Ethnic Minor Psychol. 2014;20(2):202.

20. Altschul I, Lee SJ, Gershoff ET. Hugs, not hits: Warmth and spanking as predictors of child social competence. J Marr Family. 2016;78(3):695-714.

21. Lansford JE, Sharma C, Malone PS, Woodlief D, Dodge KA, Oburu P, et al. Corporal punishment, maternal warmth, and child adjustment: a longitudinal study in eight countries. J Clin Child Adol Psychol. 2014;43(4):670-85.

22. Gershoff ET, Grogan-Kaylor A, Lansford JE, Chang L, Zelli A, Deater-Deckard K, et al. Parent discipline practices in an international sample: associations with child behaviors and moderation by perceived normativeness. Child Develop. 2010;81(2):487-502.

23. Smetana JG, Rote WM. What do mothers want to know about teens' activities? Levels, trajectories, and correlates'. J Adoles. 2015;38:5-15.

24. Mohamadirizi S, Kordi M. Association between menstruation signs and anxiety, depression, and stress in school girls in Mashhad in 2011-2012. Iran J Nurs Midwifery Res. 2013;18(5):402-7.

25. Gadade M, Patidar B, Chandane P, Chavan H. A study to assess stress experiences during the menstruation among school going adolescent girls in selected schools of Pune city. Int J Rec Scient Res. 2017;8(8):20088-90.

26. Agarwal N, Soni N, Singh SP, Soni GP. Knowledge and practice regarding menstrual hygiene among adolescent girls of rural field practice area of RIMS, Raipur (CG), India. Int J Reprod Contracept Obstet Gynecol. 2018;7:2317-21.

27. Kamath R, Ghosh D, Lena A, Chandrasekaran V. A study on knowledge and practices regarding menstrual hygiene among rural and urban adolescent girls in Udupi Taluk, Manipal, India'. Global J Med Public Health. 2013;2(4):1-9.

28. Sarkar A, Mandal R, Ghorai S. Premenstrual syndrome among adolescent girl students in a rural school of West Bengal, India. Int J Med Sci Pub Health. 2015;5(3):408.

29. Negi P, Mishra A, Lakhera P. Menstrual abnormalities and their association with lifestyle pattern in adolescent girls of Garhwal, India. J Fam Med Primary Care. 2018;7(4):804.

30. Thakre SB, Thakre SS, Reddy M, Rathi N, Pathak K, Ughade S. Menstrual hygiene: knowledge and practice among adolescent school girls of Saoner, Nagpur district. J Clin Diagn Res. 2011;5(5):102733.

Cite this article as: Vijay C, Ramesh N, Gonsalves KP. Impact of parenting style and upbringing on menstrual stress in adolescent South Indian girls. Int J Reprod Contracept Obstet Gynecol 2020;9:368896. 